\begin{tabular}{|l|l|l|l|l|l|}
\hline MUNIBE Antropologia-Arkeologia & $n^{\circ} 69$ & $257-263$ & DONOSTIA & 2018 & ISSN 1132-2217 • eISSN 2172-4555 \\
\hline
\end{tabular}

\title{
Estudio antropológico de los restos humanos hallados en el yacimiento visigodo de Riocueva (Hoznayo, Entrambasaguas. Cantabria)
}

\section{Anthropological study of the human remains from the visigothic site of Riocueva (Hoznayo, Entrambasaguas, Cantabria)}

PALABRAS CLAVES: Cueva sepulcral. Tardoantigüedad. Antopología. Paleopatología.

GAKO-HITZAK: Hilobi-kobazuloa. Antzinaro Berantiarra. Antropologia. Paleopatologia.

KEY WORDS: Cave, Late Antiquity, Anthropology, Paleopathology.

\section{Silvia CARNICERO-CÁCERES ${ }^{(1)}$}

\section{RESUMEN}

Entre los años 2011 y 2014 se llevaron a cabo distintas intervenciones arqueológicas en el yacimiento de Riocueva, un depósito en cueva de carácter sepulcral cuyos materiales han sido az.

Se presenta el estudio antropológico y paleopatológico de los restos humanos recuperados en las tres campañas. La muestra se encontraba muy fragmentada y afectada por la acción intencional del fuego. Aún así, se han podido identificar al menos 8 individuos, 4 de ellos subadultos y otros 4 adultos jóvenes, sin patologías graves o traumatismos que justifiquen su fallecimiento, sobre los que se produjeron alteraciones tafonómicas antrópicas y faunísticas (fragmentación de los huesos, dispersión y aplicación de fuego).

Tanto el yacimiento como la población muestras características similares a otros yacimientos próximos, como son las cuevas de Las Penas (Mortera, Piélagos) o La Garma (Omoño, Ribamontán del Monte).

\section{LABURPENA}

2011 eta 2014 urteen artean, Riocuevako aztarnategian hainbat lan arkeologiko egin zuten. Aztarnategiak hilobi izaera du eta bertako materialak K.a. 650 eta 730 urte artekoak dira.

Hiru kanpainetan berreskuratutako gorpuzkien ikerketa antropologikoa eta paleopatologikoa jasotzen du. Lagina oso hautsita eta suaren nahitako ekintza zela eta oso kaltetuta zegoen. Hala eta guztiz ere, gutxienez 8 banako identifikatu ahal izan dituzte; haietako 4 azpi-helduak dira, eta beste 4 heldu gazteak, heriotza justifikatuko lukeen patologia larririk edo traumatismorik gabeak. Haiengan asaldura tafonomiko antropikoak eta faunistikoak gertatu ziren (hezurren zatiketa, dispertsioa eta sua aplikatzea).

Aztarnategiak zein populazioak gertu dauden beste aztarnategi batzuetakoen antzekoak diren ezaugarriak dituzte: Las Penaseko kobazuloak (Mortera, Piélagos) edo La Garma (Omoño, Ribamontán al Monte), adibidez.

\section{SUMMARY}

From 2011 to 2014 the Mauranus Research Project team, a project whose purpose is to study the transition between the ancient and medieval world in Cantabria, carried out 3 archaeological interventions in Riocueva site. This site is a cave that corresponds to one of the entries to a karstic complex about $3 \mathrm{~km}$ long although the archaeological site occupies about 70-80m from the vestibule. Archaeological studies have confirmed the sepulchral character of the deposit, which has been dated by studying the C14 between 7th and 8th centuries AD and by TL between 6th and 9th centuries AD.

We report the anthropological and paleopathological study of the human remains recovered after the 8 archeological surveys were completed. The hole sample was very fragmented (498 human bone fragments, most of them very small), because of the action of animals and humans. Moreover, they were affected by the intentional use of fire, so all this circumstances hindered the anthropological study. The aim was to establish the Minimum Number of Individuals deposited in the cave, as well as other anthropological and paleopathological features that characterize the sample.

At least 8 individuals have been identified, 4 subadults (a perinatal, a child of 2-3 years, a child of 3-4 years and a child of 6-11 years) and 4 very young adults. No important pathologies neither traumas were recognized, so the cause of death is unidentified. Important intentional anthropogenic and faunal taphonomic alterations were reported (fragmentation of the bones, dispersion and fire damage).

Both the site and the population show similarities with other archeological sites, the caves of Las Penas (Mortera, Piélagos. Cantabria) and the Lower Gallery of La Garma (Omoño, Ribamontán del Monte. Cantabria), that we review in this report. All of them were used from 650 to 730 a.C. as atipical sepulchral sites, since the normal practice was to bury dead people in necropolis. Furthermore, the demographic profile identified, individuals who died as children subjects, subadults or very young adults, does not correspond to the pattern present in contempo-

\footnotetext{
(1) Médico Forense. Máster en Antropología Forense. Instituto de Medicina Legal de Cantabria. c/ Vargas 45 4H. 39010. Santander. Cantabria. Email: aivlisxii@yahoo.es
} 
rary necropolises, such as Santa María de Hito (Valderredible. Cantabria), El Conventón de Rebolledo (Valdeolea. Cantabria) or Santa María de Retortillo (Retortillo. Cantabria). They belong to groups of ages where mortality should decrease drastically. This can only be explained by the hypothesis of an acute infectious disease as the cause of death: a very contagious infection which causes the death of those individuals no immunized previously. In the pre-vaccination era, several of them may be candidates: smallpox, plague, typhoid fever, flu,.... In this sense, according to the hypothesis proposed by the Mauranus Project team samples have been collected to carry out studies of paleomicrobiology.

\section{INTRODUCCIÓN}

Entre los años 2011 y 2014 los arqueólogos del Proyecto Mauranus, un proyecto cuyo fin es estudiar la transición entre el mundo antiguo y medieval en Cantabria, llevaron a cabo 3 intervenciones arqueológicas en el yacimiento de Riocueva.

La cueva se corresponde con uno de los accesos a un complejo kárstico de unos $3 \mathrm{~km}$ de longitud. El yacimiento arqueológico que alberga ocupa unos 70-80m desde el vestíbulo (Gutiérrez, y Hierro, 2017). Los estudios arqueológicos han confirmado el carácter sepulcral del depósito, que ha sido datado mediante el estudio del $\mathrm{C}^{14}$ de los huesos, entre los siglos VII y VIII y mediante TL de un fragmento de cerámica, entre VI y IX d.C. (Gutiérrez y Hierro, 2017). Este uso sepulcral de cuevas en la Alta Edad Media también se ha confirmado en los yacimientos de Las Penas y la Galería Inferior de La Garma (Hierro, 2011; Arias et alii, in press).

Los trabajos arqueológicos consistieron en la realización de 8 sondeos, siendo recuperados 498 fragmentos óseos humanos, la mayoría de ellos de escaso tamaño. Muchos se encontraban afectados por el fuego. Estas alteraciones tafonómicas (figura 1), de origen antrópico e intencional, unidas a otras de tipo faunístico, que provocaron una gran dispersión y revuelto del material a lo largo de la cueva, han dificultado el estudio antropólogico.

Se ha podido determinar que en la cueva fueron depositados al menos 8 individuos, ninguno de los cuales alcanzó la plena adultez, sin que presenten patologías relevantes que justifiquen su fallecimiento.

\section{MATERIAL Y MÉTODOS}

Los restos se recibieron lavados y clasificados tras cada una de las campañas, realizándose los correspondientes informes antropológicos. Tras la última campaña, se realizó un informe final correlacionando los hallazgos de las tres intervenciones.

La reconstrucción de los restos se realizó mediante la aplicación de nitrato de celulosa (pegamento Imedio banda $a z u{ }^{\circledR}$ ), de fácil reversibilidad con acetona.

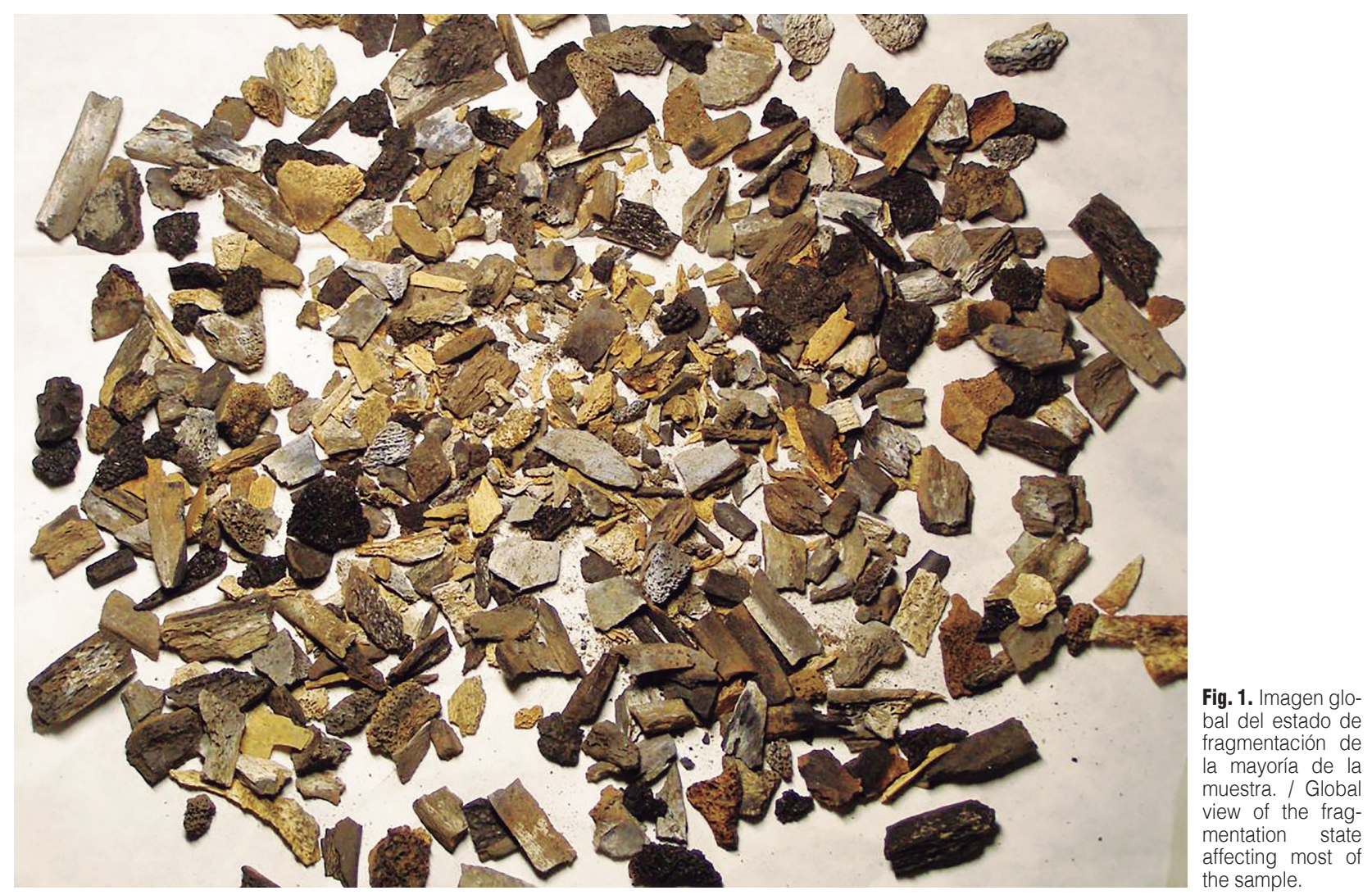


El estudio antropológico consistió en la inspección macroscópica y medición de los restos (antropometría) mediante calibre digital, cinta métrica y tabla osteométrica. Se han tomado como referencia los parámetros registrados en las RECOMENDACIONES EN ANTROPOLOGIA FORENSE de la AEAOF (2013) y los STAN$D A R S$ de Buikstra y Ubelaker (Buikstra y Ubelaker, 1994).

El objetivo principal fue identificar número mínimo de individuos (NMI) depositados en la cueva, edad aproximada en el momento del fallecimiento, sexo y cualquier patología que pudieran presentar en vida o fuera responsable de su muerte.

La edad de los individuos infantiles se ha establecido mediante la comparación de las medidas óseas y el estado de desarrollo y fusión de las epífisis con las tablas de desarrollo de Fazekas y Kósa (Fazeka Y Kósa, 1978) y Scheuer y Black (Scheuer Y Black, 2000 y 2009). En cuanto a los adultos, sólo se ha podido estimar en base a los escasos restos dentales y su desgaste (Brothwell, 1978), ya que no se han recuperado fragmentos craneales de suficiente entidad para estudiar la fusión de las suturas craneales.

En cuanto al sexo de los sujetos subadultos, si bien existen estudios como el de SCHUTKOWSKY (1993), que valora el ángulo de la escotadura ciática o fórmulas como el índice de "anchura ilíaca" de WEAVER (1980), no se ha recuperado ningún hueso de la cintura pelviana. En el caso de los adultos, la minuciosa fragmentación a la que han sido sometidos los huesos ha impedido la recuperación de huesos pelvianos o del cráneo, por lo que sólo se ha podido basar en las medidas de la cabeza radial (Robledo et alii., 2007).

Paralelamente a este estudio antropológico se está llevando a cabo un estudio de paleomicrobiología a partir de muestras dentales recogidas en este yacimiento, cuyos resultados aún son desconocidos.

\section{RESULTADOS}

Los restos, quemados o no, se encuentran distribuidos de forma irregular a lo largo diferentes sondeos sin que se puedan extraer conclusiones de su distribución.

\subsection{Número Mínimo de Individuos (NMI)}

Dada la fragmentación de la muestra, el NMI sólo se ha podido establecer a partir de dos huesos de pequeño tamaño: la vértebra C1 (atlas) y la rótula.

Se han recuperado un atlas completo de un individuo subadulto, mayor de 6 años de edad (pieza $n^{\circ}$ 168.), un hemiarco izquierdo de un infantil perinatal o menor de 2 años, un hemiarco izquierdo de otro infantil en torno a los 3-4 años y un hemiarco derecho y otro izquierdo de un individuo infantil de 2-3 años de edad. En cuanto a las rótulas, se han encontrado 4 izquierdas.
Todas ellas se encuentran completamente osificadas, por lo que pertenecen a individuos mayores de 14-16 años (edad de osificación según sea sexo femenino o masculino), si bien algunas presentan importantes diferencias de tamaño, lo que nos orientaría a individuos de mayor edad.

Por tanto, podemos asegurar que al menos fueron depositados 4 individuos infantiles y otros 4 adultos o subadultos mayores.

\subsection{Sexo}

Como ya se ha dicho, sólo se han recuperado restos de individuos adultos que puedan aportar datos sobre su sexo, ninguno de subadultos. Se trata de un cuerpo mandibular con rasgos masculinos y dos fragmentos proximales de sendos radios derechos. La cabeza radial de uno mide 19,99mm de diámetro, por lo que pertenece a un individuo femenino. El otro mide 20,59mm, aparentemente de sexo femenino, pero su longitud hace pensar que podemos encontrarnos ante un individuo masculino que aún no había completado su crecimiento.

Por consiguiente, de los 4 individuos adultos o subadultos a punto de alcanzar la madurez, uno es un varón, otro una mujer y un tercero, indeterminado.

\subsection{Edad}

Basándonos en la primera vértebra cervical, los 4 individuos subadultos presentan las siguientes edades: Perinatal, 2-3 años, 3-4 años y un infantil mayor de 6 años (probablemente adolescente).

En cuanto a los otros 4 individuos, las rótulas indican que eran mayores de 14/16 años; unas son de mayor tamaño que otras, orientándonos a individuos más maduros.

El desgaste del segundo molar de una hemimandíbula izquierda recuperada (figura 2) orienta a un indivi-

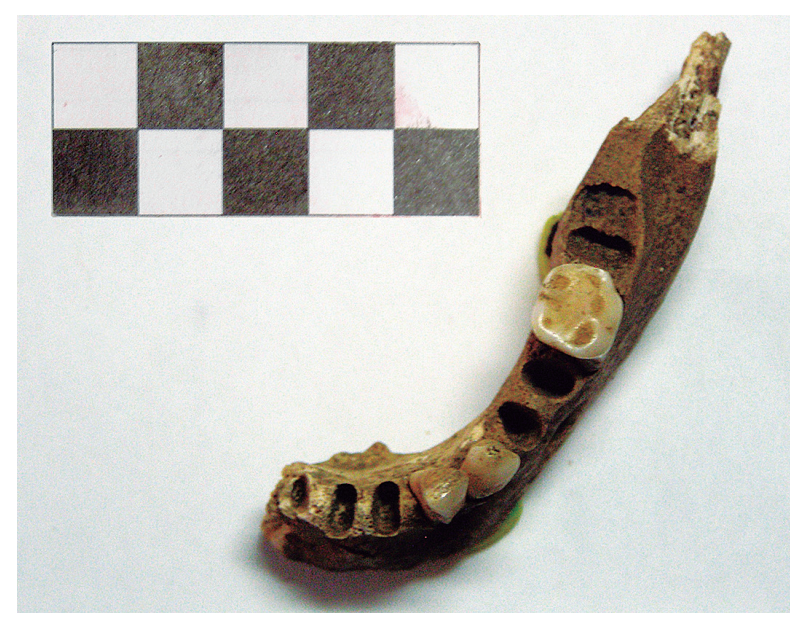

Fig. 2. Hemimandíbula izquierda. / Left hemimandible. 
duo adulto joven (17-25 años), aunque la ausencia de erupción del tercer molar indica que sería menor de 21.

El cuerpo de otra mandíbula (figura 3) conserva "in situ" el primer molar inferior derecho y los dos premolares izquierdos y, asociados a ella, 2 molares inferiores (el primer molar izquierdo y otro), 2 coronas molares muy fragmentadas, 1 incisivo, aparentemente central, 1 premolar y múltiples fragmentos de coronas y raíces. No presentan un gran desgaste, por lo que podríamos inferir un intervalo de edad similar a la mandíbula anterior.

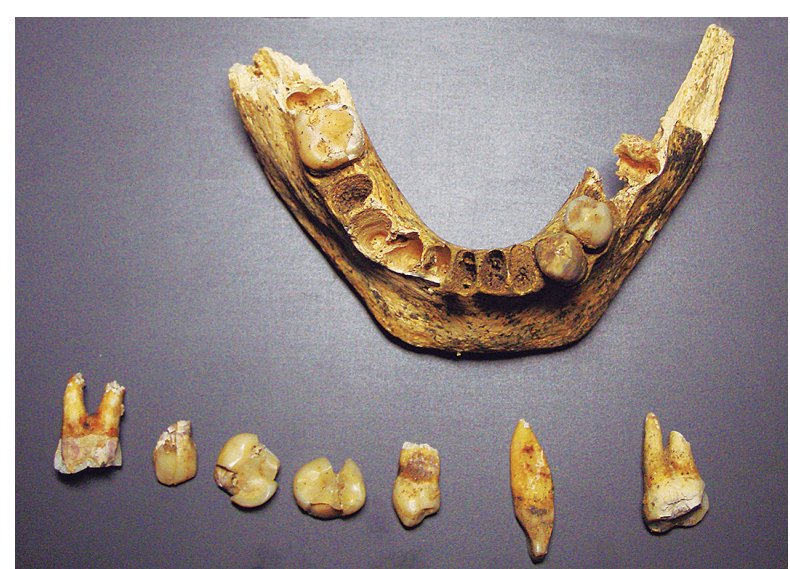

Fig. 3. Cuerpo mandibular adulto y dientes asociados, con importantes traumatismos coronales. / Adult mandibular body and associated teeth, with important coronal trauma

Se han recuperado numerosas epífisis sin fusionar, que pertenecerían a uno o varios individuos de entre 12-16 años (mujer) y 13-18 (varón), así como varios huesos pélvicos sin unir que indican que al menos uno de ellos no había alcanzado los 11 años. La dificultad de asignar estos huesos a uno o varios individuos estriba en que se trata de epífisis correspondientes a diferentes huesos y apenas existe duplicidad.

\subsection{Patologías}

No se han identificado patologías vitales o perimortales Ilamativas, aunque hay que reiterar que la muestra es muy limitada y con importantes alteraciones tafonómicas.

A nivel esquelético, 3 vértebras torácicas y una lumbar presentan marcada inserción del ligamento amarillo, variante de la normalidad, correspondiente a entesoexóstosis ligera, grado 1, tanto en la arista flava como en el proceso paraarticular (Campo, 1999) Dos de las vértebras presentan además un incipiente reborde osteofítico, grado 1 (Campo, 1999).

Como se puede ver en la figura 4, son vértebras de un adulto joven, ya que en el cuerpo vertebral aún pueden intuirse los surcos radiados propios de las vértebras juveniles. Dos costillas presentan exóstosis a nivel de la articulación costovertebral (figura 5).

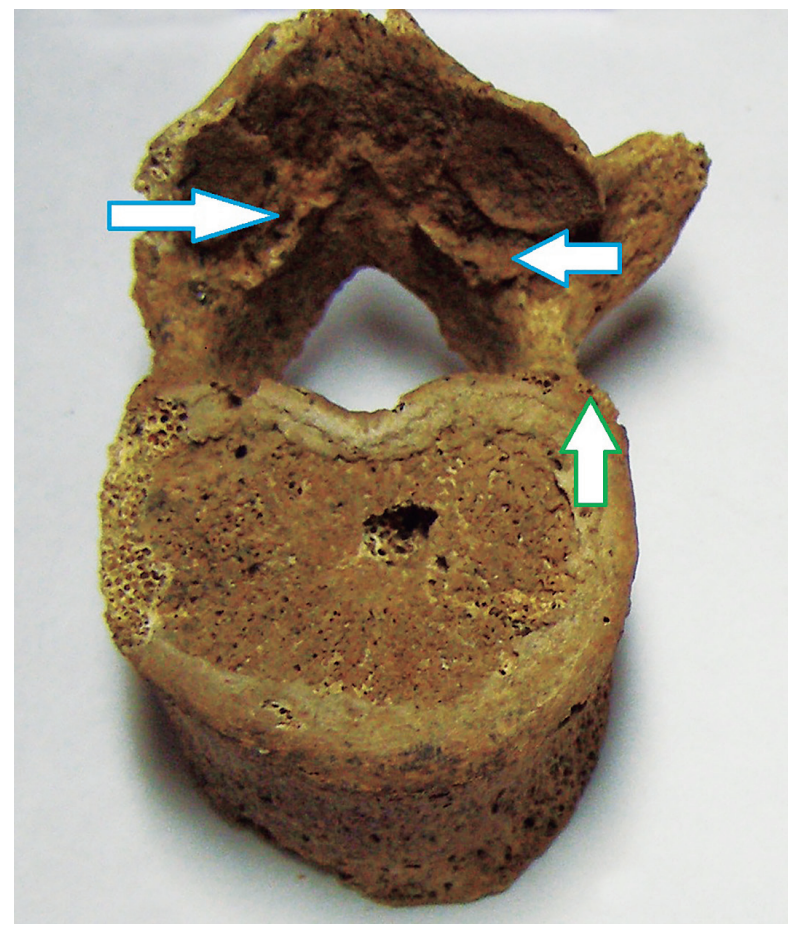

Fig. 4. Exóstosis a nivel inserción ligamento amarillo (flechas horizontales) y reborde osteofítico (flecha vertical). / Exostosis at the yellow ligament insertion (horizontal arrows) and osteophytic margin (vertical arrow).

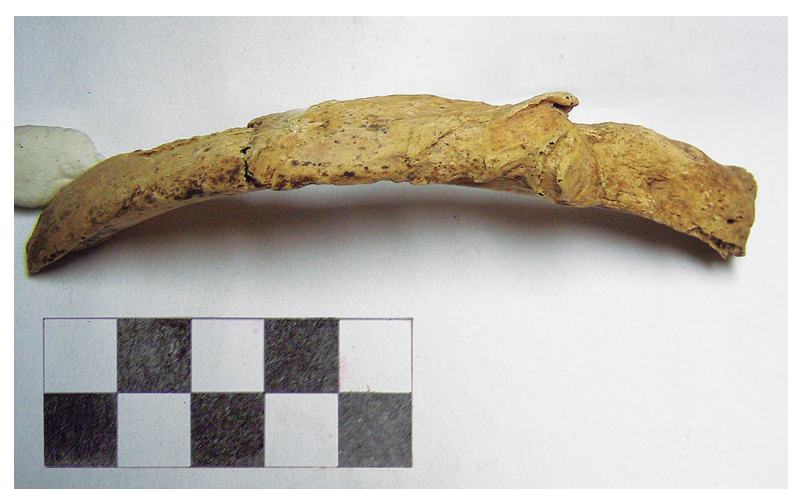

Fig. 5. Exóstosis a nivel de articulación costovertebral. / Exostosis at the costovertebral joint.

En cuanto a las piezas dentales, sólo se recuperaron 16 dientes temporales y 80 permanentes, un número significativamente bajo en relación a los, al menos, 8 individuos depositados en el yacimiento. Las causas pueden ser varias, como que el depósito original de los maxilares que las albergaban se encuentre en otra área de la cueva o que hayan sido destruídas con tanta fuerza, como ocurre con los restos identificados con el $\mathrm{n}^{\circ}$ 1510, que apenas sean reconocibles. Hay que recordar que los dientes son unas piezas de extremada resistencia, que no pueden ser alterados por los mecanismos de masticación de otros animales.

Las patologías que presentan son caries (3 piezas, una de ellas recogida en la figura 6), ligero depósito 


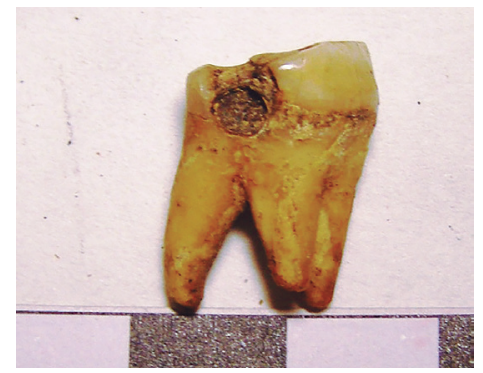

Fig. 6. Molar con caries cervical. / Molar teeth with cervical caries.

de sarro (9 piezas) y desgaste dental, muy llamativo en incisivos (en algunos casos alcanza más de la mitad de la corona). También se encontró un molar decidual con desgaste. Se han identificado débiles líneas de hipoplasia (2 piezas) y una malrotación. En general, esta población no presenta graves patologías dentales, probablemente debido, no sólo al escaso tamaño muestral, si no, sobre todo, a la edad de los individuos en el momento del fallecimiento.

\subsection{Alteraciones tafonómicas}

Una vez esqueletizados, los huesos fueron fragmentados y quemados de manera intencional; en un momento bastante posterior, los restos fueron alterados por tejones, ya que la cueva fue utilizada como madriguera.

La fragmentación de los huesos ha sido muy exhaustiva, formando fragmentos y esquirlas óseas de escaso tamaño.

Muchos de los restos han sido quemados, junto con restos de fauna, si bien los más abundantes pertenecen al cráneo. La afectación por el fuego ha sido desigual, con fragmentos de coloración grisácea-blanquecina, lo que nos indica que estuvieron expuestos directamente a la llama durante un espacio de tiempo prolongado, y otros en los que aún se mantiene parte de su coloración mientras otras áreas adquieren un tono negruzco, indicando una exposición más débil. (Figuras 7 y 8). En varios fragmentos de calota el fuego sólo ha afectado una de las tablas, unas veces la externa, otras la inter-

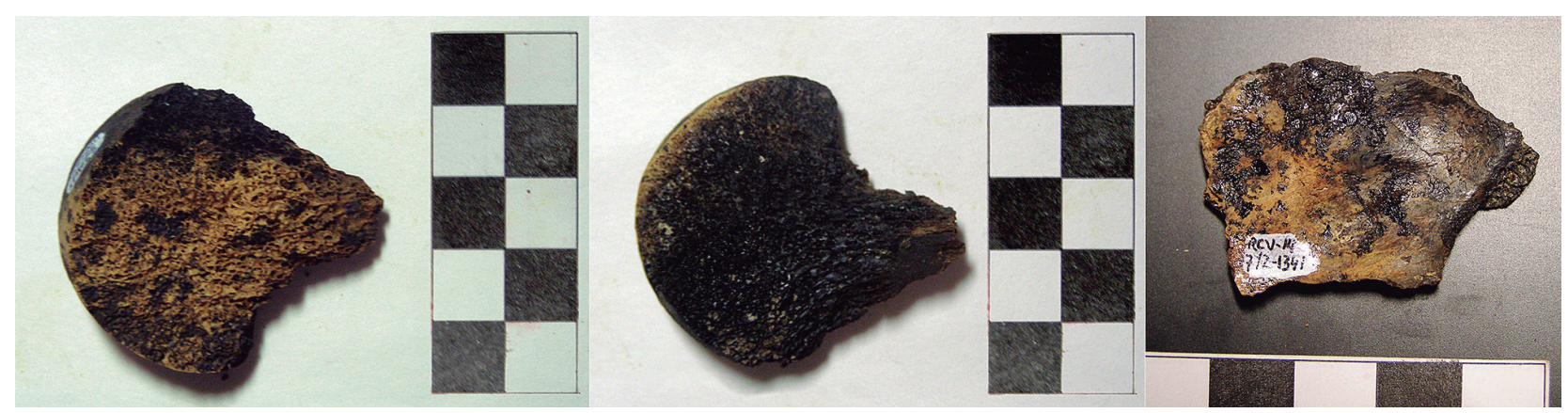

Fig. 7. Izquierda y centro: cabeza humeral fragmentada e irregularmente afectada por el fuego. Derecha: fragmento de calota. / Left and center: fragmented head humeral head and irregularly affected by fire. Right: fragment of craneal vault.
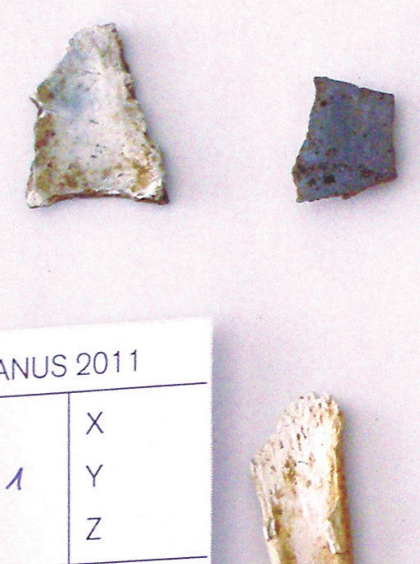

- QUEMADOS
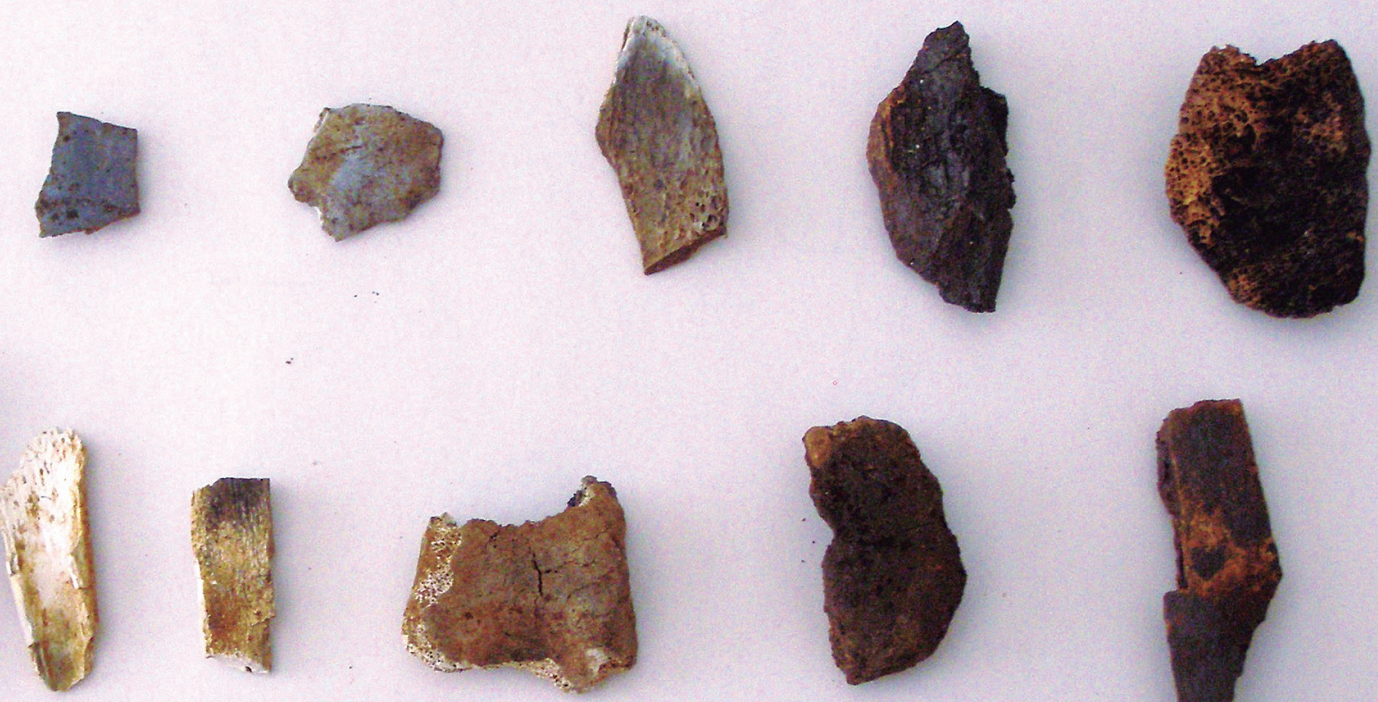

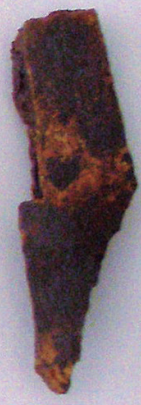

Fig. 8. Una muestra de la variabilidad de la afectación por fuego. / Colour changes produced by the fire. 
na, lo que de nuevo orienta a que fueron quemados tras su esqueletización y fragmentación intencional.

No se han encontrado marcas de carroñeo, si bien casi todas las epífisis óseas se encuentran muy erosionadas.

\section{CONCLUSIONES}

Entre el 606 y 681 d.C. se depositaron en el yacimiento de Riocueva al menos 8 individuos jóvenes. Entre los sujetos subadultos, se han encontrado restos de un individuo perinatal, un infantil de 2-3 años, un infantil de 3-4 años y un infantil de 6-11 años. Hay datos que orientan a la presencia de un juvenil/adulto joven de 1216 (mujer)/ 13-18 (varón), aunque los huesos adscritos a este individuo podrían pertenecer en realidad a dos sujetos, uno más joven y otro más cercano a la adultez (por las edades de fusión de las epífisis). Sin embargo, no disponemos de pruebas que indiquen que fueron 5 y no 4 los individuos subadultos inhumados. Una de las rótulas izquierdas halladas podría pertenecer a este último cuerpo. Además, tendríamos otros 3 sujetos en la última fase de desarrollo o que recientemente habían alcanzado la adultez. Parece probable que uno fuera un varón, otro una mujer y otro de sexo indeterminado.

Estos datos demográficos son similares a los registrados en otras cuevas donde también se ha registrado un uso sepulcral en época visigoda, como Las Penas (Mortera, Piélagos), en la que entre 666-778 se depositaron 13 individuos de entre 0 y 30-35 años (Carnicero, 2006), la Galería Inferior de La Garma (Omoño, Ribamontán del Monte), con 5 individuos entre 12 y 20 años depositados entre 688 y 754 (Arias et alii.,2018) o Cueva Foradada (Sarsa de Surta, Huesca), donde se encontraron varios individuos, la mayoría infantiles y juveniles y sólo uno mayor de 30 años (Barandiarán, 1973), como en el caso de Las Penas.

A excepción de la patología dental (3 caries, mínimo depósito de sarro y desgaste moderado), los restos estudiados no presentan signos de haber padecido enfermedades o traumatismos vitales o perimortales, si bien el escaso porcentaje de conservación de los esqueletos impide descartar con seguridad su existencia. Además, al igual que ocurre en el yacimiento de Las Penas o La Garma, no existen datos físicos que expliquen la causa de muerte de todos estos individuos. El desgaste que presentan los dientes, similar al de otros yacimientos de la época, nos hace pensar en que estos individuos tenían una dieta rica en elementos bastante abrasivos (probablemente harina de cereales mezclada con minerales desprendidos de los molinos usados).
Tras su esqueletización, los huesos fueron fragmentados de manera intencional. Si bien no se han encontrado elementos en la cueva que hayan podido ser usados con este fín, como afortunadamente ocurre en el caso de La Garma, el grado y extensión de la fragmentación que presentan no corresponden a eventos fortuitos o a la acción de los animales, si bien éstos han tenido un papel muy importante en la dispersión de los restos. Esto implica que no se han podido recuperar huesos en conexión anatómica o en una posición que oriente su pertenencia a un mismo individuo, como sí ocurre en Las Penas o La Garma. Al contrario que en la cueva de Las Penas, donde los cráneos fueron depositados en una galería diferente y allí fueron fragmentados y quemados, o en La Garma, donde los cráneos se fragmentaron in situ, y ningún hueso aparece afectado por el fuego, en Riocueva casi todos los huesos del esqueleto han sido reducidos a pequeños fragmentos, de manera que apenas existen huesos largos completos.

Muchos de los huesos fueron quemados años después del depósito, una vez descarnados y secos, como la mayoría de los cráneos de Las Penas. En el caso de Riocueva, a diferencia de aquél, es que los huesos no sólo pertenecen a la calota, ya que también se han recuperado huesos de las manos y los pies o incluso diáfisis de huesos largos, mezclados con abundante fauna a la que también afectó el fuego. Otra particularidad de Riocueva es que los restos humanos quemados no se localizan en un área concreta, aunque, debido a la acción de dispersión llevada a cabo por los tejones durante años no podemos descartar que en un principio sí que se dispusieran de una manera concreta.

La edad y causa de muerte de estos individuos sigue siendo un misterio. El perfil demográfico identificado, individuos que fallecieron siendo sujetos infantiles, subadultos o adultos muy jóvenes, no se corresponde con el patrón presente en necrópolis coetáneas, como Santa María de Hito (Galera, 1989), El Conventón de Rebolledo o Santa María de Retortillo (en estudio por la autora), por no hablar de otras más alejadas geográficamente. En todas ellas están bien representados todos los grupo etarios, obteniéndose las típicas curvas de mortalidad en $U^{1}$, propias de poblaciones preindustriales. Sin embargo, en estos yacimientos no hay sujetos adultos y seniles, individuos que, por ley natural, deben fallecer en mayor número. Sin embargo abundan aquellos que pertenecen a unos grupos de edades donde la mortalidad debería disminuir drásticamente. Esto sólo puede explicarse mediante la hipótesis de una enfermedad infecciosa aguda como causa de su muerte. Una infección bastante contagiosa, que produce la muerte de aquellos individuos que no se han inmunizado a ella

\footnotetext{
${ }^{1}$ Esta curva presenta dos picos de aumento de la mortalidad correspondientes a dos épocas concretas: la primera infancia, caracterizada por una mayor debilidad del ser humano y susceptibilidad de fallecer por diversas enfermedades y peligros, y los adultos maduros. Estas curvas suelen invertirse cuando una sociedad atraviesa un conflicto bélico, por ejemplo, de modo que el pico de mortalidad se da en el tramo de los subadultos y adultos jóvenes, o durante brotes epidémicos cuando una enfermedad afecta a las nuevas generaciones no inmunizadas.
} 
por no haber tenido contacto con la misma previamente. En la era prevacunación, varias pueden ser las candidatas: viruela, peste, fiebre tifoidea, gripe,...

En este sentido, en apoyo a la hipótesis planteada por el equipo de Proyecto Mauranus (Hierro, 2011), se han recogido muestras para realizar estudios de paleomicrobiología. En el año 2013 la autora tomó muestras de La Garma, Los Goros y Las Penas y se realizó un estudio de colaboración entre los servicios de Anatomía Patológica (Departamento de Patología Molecular) y Microbiología del Hospital Universitario Marqués de Valdecilla. Lamentablemente, los resultados, presentados en el XII Congreso de la Sociedad Española de Paleopatología (Cuenca), no fueron concluyentes. Con posterioridad, se han remitido muestras de los yacimientos de Riocueva, La Garma y Las Penas para su estudio en el laboratorio Paleogenetics, de la Universidad de Tübingen (Alemania), con el fin de confirmar la hipótesis.

A la espera de futuros estudios que aclaren las circunstancias de la muerte de estos individuos, sólo podemos concluir que Riocueva se une a los múltiples yacimientos de depósito sepulcral en cueva de época visigoda que salpican la geografía española y el sur de Francia en los que se ocultaron los cuerpos de unos individuos jóvenes, que murieron por causas naturales y probablemente infecciosas.

\section{BIBLIOGRAFÍA}

Arias, P., Gutierrez Cuenca, E., Hierro Gárate, J.A., Etxeberria, F., Herrasti, L., Uzquiano, P., 2018. Hidden in the depths, far from people: Funerary activities in the Lower Gallery of La Garma and the use of natural caves as burial places in early medieval Cantabria, northern Spain. In: Bergsvik, K. A., Dowd, M. (ed.), Caves and ritual in Medieval Europe, AD 500-150, 132-151. Oxbow books.

Barandiarán, I., 1973. Restos visigodos en la cueva Foradada (Sarsa de Surta, Huesca). In: Estudios de Edad Media de la Corona de Aragón, IX. Zaragoza: Consejo Superior de Investigaciones Científicas-Escuela de Estudios Medievales, 9-48.

Buikstra, J.E., Ubelaker, D.H., 1974. Standards for data collection from human skeletal remains. Arkansas Archeological Survey. Fayetteville.

Campo, M., 1999. El babel terminológico: A vueltas con las lesiones elementales. Boletín de la Asociación Española de Paleopatología 23, 2-3.

Carnicero Cáceres, S., 2006. Estudio antropológico del yacimiento arqueológico "Cueva De Las Penas" (Mortera, Piélagos, Cantabria). Sautola 12, 295-300.

Fazekas i, Kósa F., 1978. Forensic Fetal Osteology. Akadémiai Kiadó. Budapest.

Galera, V., 1989. La población medieval cántabra de Santa María de Hito. Aspectos Paleobiodemográficos, Morfológicos, Paleopatológicos, Paleoepidemiológicos y de Etnogénesis. [Tesis doctoral]. Alcalá de Henares. Universidad de Alcalá.

Gutierrez Cuenca, E., Hierro Gárate, J.A., Paredes Courtot, H., 2017. Cerámica común de cocina de los siglos VII-VIII en contexto funerario. La cueva de Riocueva (Cantabria). Boletín Ex Officina Hispana 8 (marzo), 100-103.
Gutierrez Cuenca, E, Hierro Gárate, J.A., 2016. Desenterrando a los últimos visigodos. Actuaciones arqueológicas en Riocueva (2010-2014). En : Cantabria. Nuevas evidencias arqueológicas, 157-185. ADIC-Los Cántabros, Santander.

Hierro Gárate, J.A., 2011. La utilización sepulcral de las cuevas en época visigoda: los casos de las Penas, la Garma y el Portillo del Arenal (Cantabria). Munibe Antropologia-Arkeologia $62,351-402$.

Lewis, M., 2018. Paleopathology of Children. Identification of Pathological Conditions in the Human Skeletal Remains of Non-Adults. Academic Press. Elsevier.

Robledo, M.M., Sánchez, J.A., Pumar, M., Labajo, E., 2007. Determinación del sexo según el diámetro de la cabeza del radio. Revista de la Escuela de Medicina Legal (Sep), 43-51.

Scheuer, L., Black, S., 2000. Developmental Juvenile Osteology. Academic Press; Elsevier.

Schaefer, M., Black, S., Scheuer, L., 2009. Juvenile Osteology. A laboratory and field manual. Academic press. Elsevier. 
